\title{
Excision Margins of Melanoma Make a Difference: New Data Support an Old Paradigm
}

\author{
Merrick I. Ross ${ }^{1}$ and Charles M. Balch ${ }^{2}$ \\ ${ }^{1}$ Department of Surgical Oncology, University of Texas MD Anderson Cancer Center, Houston, TX; ${ }^{2}$ Division of Surgical \\ Oncology, Department of Surgery, University of Texas Southwestern Medical Center, Dallas, TX
}

The surgical standard for the local treatment of a primary melanoma is a wide excision of the diagnostic biopsy site and/ or residual intact primary lesion, inclusive of a surrounding margin of normal skin, en bloc with the underlying subcutaneous tissue including the superficial fascia. The rationale for such a surgical approach is to excise both the primary melanoma and any surrounding microsatellites in the lymphatics. Failure to excise both the primary and subclinical locally metastatic disease could result in a local recurrence (LR), which in reality is better termed "local metastasis." Such events have been associated with melanoma mortality; therefore, the concern of inadequate surgery leading to LR and a risk of dying has been the underpinnings of the widely adopted paradigm that $L R$ is both a function of primary tumor biology (tumor thickness and ulceration in particular) and extent of excision margin and that wider margins would be required for high-risk tumors. ${ }^{1-3}$ If such a paradigm were true, then various outcome measures, including the frequency of local and regional recurrences, and possibly overall survival, would be impacted by the extent of excision margin, and in turn, the following assumptions or even conclusions could be made: (1) microscopic satellite disease is present more frequently with thicker (higher risk) melanomas and may have spread further from the periphery of the primary melanoma; (2) these microsatellites are a source of subsequent locoregional and distant relapse; and (3) wider margins more completely remove microscopic disease that would otherwise remain if narrower margins were used. This paradigm was tested formally in multiple, prospective,

(C) Society of Surgical Oncology 2015

First Received: 19 October 2015;

Published Online: 11 November 2015

C. M. Balch

e-mail: charles.balch@UTSouthwestern.edu randomized, surgical trials across the entire spectrum of stage I and II thickness groups, comparing conservative margins (1 or $2 \mathrm{~cm}$ ) to wider margins $(3,4$, or $5 \mathrm{~cm}) .{ }^{4}$ Long-term results of these trials failed to demonstrate that a wider excision independently predicted melanoma-specific survival. ${ }^{4}$ One could conclude, and many have, that there is little evidence to support the paradigm and believe that any width of histologically clear excision margin is acceptable.

However, the prospectively randomized trial from the United Kingdom Melanoma Study Group (UKMSG) did demonstrate that the combined frequency of local, in transit, and nodal relapses were significantly higher when $1-\mathrm{cm}$ margins compared with when $3-\mathrm{cm}$ margins were used to treat 900 patents with melanomas thicker than $2 \mathrm{~mm}$ (T3 and T4). ${ }^{5}$ In the World Health Organization trial for T1 and T2 melanomas, a trend was observed for an increased incidence of LR in the subset of patients with T2 melanomas when randomized to receive the narrower 1-cm margin compared with a $3-\mathrm{cm}$ margin. ${ }^{6}$ It is important to note that no study has prospectively examined margins narrower than $1 \mathrm{~cm}$ for invasive melanoma of any thickness. Furthermore, none of these trials included extensive analyses of the wide excision specimens looking for the presence of microsatellite disease.

Recent reports have suggested, for the first time, that there is some risk for using too conservative margins when excising primary melanomas, not only in terms of LR rates but also in terms of melanoma-specific mortality. This includes two reports in this issue of the Annals of Surgical Oncology for thin (T1) and intermediate thickness (T2) melanomas as part of a series of reports for various T-stage patients from the Melanoma Institute of Australia (MIA) as well as a recent update from the aforementioned UKMSG trial presented at the 2015 annual meeting of the American Society of Clinical Oncology. ${ }^{71}$ In a recent update of the UKMSG Trial, with a mean follow-up of 8.8 years, patients with a narrower 1-cm margin had a higher melanoma-specific 
death rate $(p=0.039)$ and a worse melanoma-specific survival rate $(p=0.05)$ compared with those having a $3-\mathrm{cm}$ surgical margin. In a multivariate analysis, surgical margin was an independent predictor of a worse outcome $(p=0.036) .{ }^{11}$

Additional evidence about the importance of surgical margins comes from the above-mentioned publications from the MIA, where they retrospectively queried their melanoma database, the largest of its kind in the world, to determine outcomes of large patient groups with various thresholds of melanoma thickness. The two most recent of these publications, in this issue of the Annals of Surgical Oncology, reported on LR rates and survival outcomes among 11,290 patients with thin melanomas ( $\mathrm{T} 1$ or $\leq 1.0$ $\mathrm{mm}$ thickness) and 2131 patients with $\mathrm{T} 2$ melanomas with thicknesses of $1.0-2.0 \mathrm{~mm} .^{7,8}$ They specifically examined the pathological specimens with the assumption that a 1.0$\mathrm{cm}$ surgical margin used in the operating room was equivalent to a $0.8-\mathrm{cm}$ margin measured after fixation in the pathology suite (i.e., $20 \%$ shrinkage factor).

Among the 11,290 T1 melanomas, there were 176 patients with a LR after a median follow-up of 98 months, for a $1.56 \%$ LR rate (which is probably the largest number reported in the literature to date). LR was associated with narrower margins [hazard ratio (HR) $=0.95 ; p=0.001$ ], as well as the histological type (desmoplastic, acral, and lentigo maligna melanomas) $(p=0.005)$. They concluded that a surgical margin should be $\geq 1.0 \mathrm{~cm}$ where possible to reduce the risk of LR, especially for patients with desmoplastic, acral, and lentigo maligna melanomas. ${ }^{7}$

Among the $2131 \mathrm{~T} 2$ patients, 326 patients (15\%) had undergone an excision $<8 \mathrm{~mm}^{8}$ Patients who had a pathologic excision margin $<8 \mathrm{~mm}$ (equivalent to $1 \mathrm{~cm}$ in vivo) had poorer prognosis in terms of disease-free survival compared with the 8 - to $16-\mathrm{mm}$ group (equivalent to $1-2 \mathrm{~cm}$ in vivo; $p=0.044$ ). Pathologically determined excision margins $<8 \mathrm{~mm}$ were associated with worse regional node recurrence-free survival and distant recurrence-free survival compared with margins $\geq 8 \mathrm{~mm}$ ( $p=0.049$ and $p=0.045$, respectively). In a multivariate analysis, both width of excision and tumor thicknesses were the only independent predictors of LR. Based on the clinical-pathological features, no obvious differences in inherent biology exists between the two groups that could explain any differences in outcome and therefore provides some credibility to the multivariate analyses. However, these results failed to translate into a statistically significant difference in melanoma-specific survival. When comparing $8-\mathrm{mm}$ with $16-\mathrm{mm}$ pathologic margins (i.e., $1-\mathrm{cm}$ vs. $2-\mathrm{cm}$ surgical margins), no differences were observed in any of the survival outcomes. Only the deep margin of $<8 \mathrm{~mm}$ proved to be an independent predictor of local and intransit recurrence-free survival $(p=0.003)$ in all excision margin categories. ${ }^{8}$
A previously reported study by the MIA evaluated the extent of peripheral margins in 2681 patients with $\mathrm{T} 2$ melanomas $<2 \mathrm{~mm}$ in thickness, also concluded that surgical margins $<1 \mathrm{~cm}$ may be too narrow due to an associated higher LR rate. ${ }^{12}$ With a median follow-up of 83.9 months, the LR incidence was $2.9 \%$. In multivariate analysis, both the width of excision and the tumor thicknesses were the only independent predictors of LR. When patients with less than 1-cm margins were excluded, margin width was no longer an independent predictive of LR. ${ }^{12}$

In two other recently published papers, the MIA reported the outcomes and LR rates of 1587 patients with T3 melanomas (2.1- to 4.0-mm thickness) and 632 patients with T4 melanomas (>4.0-mm thickness). ${ }^{9,10}$ Among the T3 melanoma patients, a histopathologic excision margin of $8 \mathrm{~mm}$ or more (i.e., equivalent to $\geq 1-\mathrm{cm}$ surgical margin) was associated with increased local and in-transit recurrence-free survival ( $\mathrm{HR}=0.54 ; p=0.008)$ and disease-free survival $\quad(\mathrm{DFS}) \quad(\mathrm{HR}=0.59 ; \quad p=0.001)$ compared with a $<8$-mm margin. ${ }^{9}$ The $<8$-mm group also had reduced distant recurrence-free survival (DRFS) compared with the 8- to $16-\mathrm{mm}$ group $(\mathrm{HR}=1.63$; $p=0.038){ }^{9}$ Among the T4 melanomas, an excision margin as a continuous variable was a significant predictor of local (HR, 0.91; $p<0.001)$ and locoregional (HR, 0.97; $p=0.042$ ) tumor control in a multivariate analyses. ${ }^{10}$ Patients with histopathologic margins $\leq 1.6 \mathrm{~cm}$ (i.e., equivalent to a $2.0-\mathrm{cm}$ surgical margin) also had worse local disease-free survival (HR, $2.41 ; p=0.01) .{ }^{10}$

The Australian group concluded that surgical margins $<1.0 \mathrm{~cm}$ for T1 and T2 melanoma may be too narrow and that a surgical margin $<2.0 \mathrm{~cm}$ for T3 and T4 melanomas may be too narrow because of the higher rate of LR. Their evidence supports current recommendations to excise even the earliest presentation of invasive melanomas with a surgical margin no less than $1.0 \mathrm{~cm}$ and is consistent with the findings in a subsequently published meta-analysis. ${ }^{13}$ However, neither study demonstrated any association of margin width and melanoma-specific survival.

Despite the large sample size of patients, these were retrospective analyses and conclusions that compare nonrandomized groups are always suspect, as unknown prognostic factors and surgeon judgement in selecting treatment options could not be accounted for. They did not report whether or not intralymphatic disease (microsatellites) were identified, but of course this would depend on extensive serial sectioning of the pathological specimen. Interestingly, in reports about patients in whom microsatellites were identified, the prognosis was poor. ${ }^{14,15}$ One unexpected finding from the analysis of the patients with T2 melanomas is that a deep surgical margin $<8 \mathrm{~mm}$ (or $1.0 \mathrm{~cm}$ in vivo) was independently associated with worse outcomes, which to our knowledge has never before 
been reported. ${ }^{8}$ While interesting, we are skeptical about such a finding because of a variety of differences between patients not controlled for, such as percent body fat, the amount of adipose tissue in different anatomic locations, and whether or not the surgical excision extended to the underlying muscular fascia. Currently, there are no prospective data to answer this question and, as a result, there are varying expert recommendations. ${ }^{16}$ Unfortunately, the large, prospective trials conducted to date have not standardized the actual depth of excision.

Even though a LR is an uncommon event, it is widely viewed as a benchmark of surgical quality control. LR is most likely a manifestation of residual microsatellites in the immediate area left behind after surgery and is associated with very poor overall survival, similar to that of intralymphatic metastases (in transits or satellites). ${ }^{1-3,14}$ This explanation provides a logical rationale for performing a wide excision of normal-appearing skin surrounding a primary melanoma; that is, to excise microsatellite metastases that are usually not detected without serial sectioning of the excised primary melanoma and adjacent skin.

Despite the retrospective nature of the MIA analyses, these reports, combined with the update of the UKMSG, have provided additional evidence that affirm the following concepts: (1) a LR is a function of both biology of the primary tumor and extent of surgical excision; (2) thin melanomas have a low risk for recurrence and narrow $(1 \mathrm{~cm})$ margins are safe (but not less than $1.0 \mathrm{~cm}$ ); (3) thicker and/or ulcerated melanomas are associated with a higher incidence of clinically occult intralymphatic metastases that can be removed with wider excisions, in turn lowering the risk of subsequent local and/or regional recurrences; and (4) increased rates of local and regional failure can have an impact on survival.

\section{CONCLUSIONS}

These recent publications are consistent with current guidelines and recommendations about the width of surgical margins that are tailored to melanoma thickness. These results about surgical outcomes remind us that we should not be cavalier about excision margins and that inadequate width or depth can contribute to an unfavorable outcome, especially since the recommended $1-$ or $2-\mathrm{cm}$ margins can be closed primarily and result in little early or late surgical morbidity, except in a few anatomically restricted areas, such as the face, hands, and feet. Simply stated, for melanomas $<$ and $>2 \mathrm{~mm}, 1-\mathrm{cm}$ and $2-\mathrm{cm}$ margins respectively appear to be wide enough, with still some lingering uncertainty about the appropriate margin in 1- to 2-mm subgroup. A recently opened international, prospective, randomized trial of $1-\mathrm{cm}$ versus $2-\mathrm{cm}$ margins for melanomas thicker than $2 \mathrm{~mm}$ should add some clarity. No data exist that any margin wider than $2 \mathrm{~cm}$ (i.e., 3, 4, or $5 \mathrm{~cm}$ ) would result in any superior disease-specific outcomes, but these wider margins are associated with increased surgical morbidity. The evidence is weaker about the depth of excision, and therefore we believe that the current practice of incorporating a depth of subcutaneous tissue inclusive of the superficial fascia is sufficient without attempting to achieve a specifically measured deep margin of $1.0 \mathrm{~cm}$. We do congratulate the efforts by the UKMSG Trial investigators for persisting in long-term evaluations of their randomized melanoma patients, and the MIA investigators in carrying out rather extensive analyses of a very large database. Taken together, these studies provide us with compelling data that support the paradigm from which we conduct our surgical practice.

\section{REFERENCES}

1. Balch CM, Soong SJ, Smith T, Ross MI, Urist MM, Karakousis $\mathrm{CP}$, et al. Long-term results of a prospective surgical trial comparing $2 \mathrm{~cm}$ vs. $4 \mathrm{~cm}$ excision margins for 740 patients with 14 mm melanomas. Ann Surg Oncol. 2001;8:101-8.

2. Urist MM, Balch CM, Soong S, Shaw HM, Milton GW, Maddox WA, et al. The influence of surgical margins and prognostic factors predicting the risk of local recurrence in 3445 patients with primary cutaneous melanoma. Cancer. 1985;55:1398-402.

3. Karakousis CP, Balch CM, Urist MM, Ross MM, Smith TJ, Bartolucci AA. Local recurrence in malignant melanoma: longterm results of the multiinstitutional randomized surgical trial. Ann Surg Oncol. 1996;3:446-52.

4. Ross MI, Gershenwald JE. Evidence-based treatment of earlystage melanoma. J Surg Oncol. 2011;104(4):341-53.

5. Thomas JM, Newton-Bishop J, A'Hern R, Coombes G, Timmons $\mathrm{M}$, Evans J, et al. Excision margins in high-risk malignant melanoma. N Engl J Med. 2004;350:757-66.

6. Cascinelli N. Margin of resection in the management of primary melanoma. Semin Surg Oncol. 1998 14:272-5.

7. MacKenzie Ross AD, Haydu LE, Quinn MJ, et al. The association between excision margins and local recurrence in 11,290 thin (T1) primary cutaneous melanomas. A case-control study. Ann Surg Oncol. 2015. doi:10.1245/s10434-015-4942-0.

8. Haydu LE, Stollman JT, Scolyer RA, et al. Minimum safe pathologic excision margins for primary cutaneous melanomas (1-2 mm in thickness): analysis of 2131 patients treated at a single center. Ann Surg Oncol. 2015. doi:10.1245/s10434-0154575-3.

9. Lamboo LG, Haydu LE, Scolyer RA, Quinn MJ, Saw RP, Shannon KF, et al. The optimum excision margin and regional node management for primary cutaneous T3 melanomas (2$4 \mathrm{~mm}$ in thickness): a retrospective study of 1587 patients treated at a single center. Ann Surg. 2014;260(6):1095-102.

10. Pasquali S, Haydu LE, Scolyer RA, Winstanley JB, Spillane AJ, Quinn MJ, et al. The importance of adequate primary tumor excision margins and sentinel node biopsy in achieving optimal locoregional control for patients with thick primary melanomas. Ann Surg. 2013;258(1):152-7.

11. Hayes AJ, Maynard L, A'Hern R, et al. Long-term follow-up of survival in a randomised trial of wide or narrow excision margins in high risk primary melanoma. J Clin Oncol. 2015;33(suppl; abstr 9001). 
12. McKinnon JG, Starritt EC, Scolyer RA, McCarthy WH, Thompson JF. Histopathologic excision margin affects local recurrence rate, analysis of 2681 patients with melanomas $<2$ mm thick. Ann Surg. 2005;241:326-33.

13. Sladden MJ, Balch C, Barzilai DA, et al. Surgical excision margins for primary cutaneous melanoma. Cochrane Database Syst Rev. 2009;4(4):CD004835.

14. Balch CM. Microscopic satellites around a primary melanoma: another piece of the puzzle in melanoma staging. Ann Surg Oncol. 2009; 16:1092-4.
15. Kimsey TF, Cohen T, Patel A, Busam KJ, Brady MS, et al. Microscopic satellitosis in patients with primary cutaneous melanoma: implications for nodal basin staging. Ann Surg Oncol. 2009;16:1176-83.

16. Grotz TE, Markovic SN, Erickson LA, Harmsen WS, Huebner M, Farley DR, et al. Mayo Clinic consensus recommendations for the depth of excision in primary cutaneous melanoma. Mayo Clin Proc. 2011;86:522-8. 